\title{
Asymptotic Problems - from Control Systems to Semigroups
}

\author{
Fritz Colonius* Wolfgang Kliemann ${ }^{\dagger} \quad$ Luiz A. B. San Martin ${ }^{\ddagger}$
}

December 15, 2021

\section{Introduction}

Semigroups of operators or of homeomorphisms show up in a natural way in many areas in mathematics, such as dynamical systems (one parameter groups of homeomorphisms), Markov processes (one parameter groups of operators), control theory (control semigroups), or (random) skew product flows (measurable or continuous families of diffeomorphisms connected by one parameter groups of shifts). In all these examples the semigroups depend on a real parameter, usually interpreted as time, which allows to study their asymptotic behavior and consequently their dynamic characterization, to various degrees of completeness. In the 'abstract' setting of semigroup actions on a state space, such a parameter is not available. However, several basic dynamical concepts, such as orbits, regions of transitivity, etc. can be formulated for general semigroup actions. Recently, also the idea of chain transitivity has been studied for general classes of semigroups, Barros/San Martin [2].

This paper outlines some ideas for an asymptotic or dynamic theory of semigroup actions. To this end we briefly describe the basic settings for two prototypical areas: dynamical systems and control theory. In the theory of dynamical systems, the basic objects are flows given by one parameter families of diffeomorphisms. Their asymptotic analysis is developed around the ideas of transitivity, chain transitivity, linearization (with its two components on fiber bundles and

\footnotetext{
*Institute for Mathematics, University of Augsburg, 86135 Augsburg/Germany, colonius@math.uni-augsburg.de

${ }^{\dagger}$ Department of Mathematics, Iowa State University, Ames Iowa/USA, kliemann@iastate.edu

${ }^{\ddagger}$ Instituto de Matematica, Universidade Estadual de Campinas, Cx. Postal 6065, 13.081-970 Campinas-SP, Brasil, smartin@ime.unicamp.br
} 
associated linear cocycles), and ergodic theory. Control systems can be viewed as dynamical systems over a shift space of admissible control functions, leading to a skew product flow on the state space. For these control flows the theory of dynamical systems is used to study their asymptotic behavior. However, when viewed as control systems on a state space, the flow property is lost and the basic concepts appear as objects of the control semigroup (control sets, chain control sets and their order, spectral intervals, etc.). Sections 2. and 3. give an outline of this approach to dynamical systems and to control theory. In Section 4. we interpret the basic setting of control theory in the language of semigroups and their actions. Emphasis is placed on bilinear control systems in $\mathbb{R}^{d}$ and its semigroup analogue, the action of subsemigroups of $S l(d, \mathbb{R})$ on flag manifolds.

Several important concepts for control semigroups are easily generalized to abstract semigroups, such as orbits or control sets. These concepts do not need an explicit parameter 'time'. Semigroups with an additional structure, defining a neighborhood of 'infinity' also allow for the idea of chain control sets and their analysis with respect to control sets. This circle of ideas is described in Section 5., together with the resulting problems of characterizing semigroups with respect to their (chain) transitivity behavior. This leads naturally to a concept of bifurcations of semigroups. As we will see, several standard topics in semigroup theory (invariance, compression semigroups, compactification, algebraic characterizations, in particular, for subsemigroups of semi-simple Lie groups) enter into this discussion.

The last section outlines some ideas on topological classification. Such a classification, based on topological equivalence or conjugacy of systems, is currently available for hyperbolic linear dynamical systems and for hyperbolic linear random flows. Both require the study of the compact component (invariant subspaces) and of the associated linear cocycle (Lyapunov exponents).

\section{Dynamical Systems}

The basic considerations for the study of global and asymptotic behavior are best explained for dynamical systems, since this theory has made numerous advances during the last decades. We will discuss some of the underlying ideas, keeping in mind the possibility of generalizations to control systems, random systems, and eventually to semigroups. The presentation here concentrates on the continuous time case (i.e., on flows induced by vector fields), but the basic ideas are similar for discrete time systems (i.e. for flows induced by iterations of maps).

Let $M$ be a finite dimensional (sufficiently smooth) manifold and denote by 
$\mathfrak{X}(M)$ the vector fields on $M$. In this paper we only discuss complete vector fields (i.e., the solutions exist for all times $t \in \mathbb{R}$ ), which holds true for all $X \in \mathfrak{X}(M)$, e.g., if $M$ is compact. A dynamical system is then a flow of homeomorphisms or even diffeomorphisms

$$
\Phi: \mathbb{R} \times M \rightarrow M
$$

where $\Phi(t, x)$ is the solution of $\dot{x}=X(x)$ with initial value $x=\Phi(0, x) \in M$. The goal now is to study the space $\mathfrak{X}(M)$ via the behavior of the associated flows.

For an individual vector field $X \in \mathfrak{X}(M)$ the analysis of the flow (1) could be accomplished in the following steps:

1. Find the limit sets $\omega(x)$ and $\omega^{*}(x)$ for $x \in M$. Here the positive limit set $\omega(x)$ is defined as $\omega(x)=\left\{y \in M\right.$, there exists a sequence $t_{k} \uparrow \infty$ such that $\left.y=\lim _{k \rightarrow \infty} \Phi\left(t_{k}, x\right)\right\}$, and similarly for the negative limit set $\omega^{*}(x)$ using sequences $t_{k} \downarrow-\infty$.

2. Determine the behavior of (1) on limit sets which includes the study of transitivity, mixing, invariant measures, chaos, etc.

3. Analyze the flow (1) around the limit sets, e.g. attraction and repulsion, or via linearization and Lyapunov exponents.

4. Combine the results of (i) - (iii) into a global picture.

Instead of studying individual dynamical systems $X \in \mathfrak{X}(M)$, one often imbeds the flow (1) into a family of systems $\Phi^{\alpha}$, with $\alpha$ in some index set, studies the regular elements of this family, and tries then to characterize the 'pathological' cases by comparison with the regular ones. Typical families are continuous paths, where the index set is a (multidimensional) interval in $\mathbb{R}^{p}, p \geq 1$, which leads to the approach of bifurcation theory, or a neighborhood of $X$ in the Whitney $C^{k}$-topology of $\mathfrak{X}(M)$ with $k \geq 1$, which leads to the approach of structural stability. Recently, time varying families of vector fields have also been considered in a deterministic or stochastic context, compare Sections 3. and 6. below. For low dimensional systems with simple limit sets (fixed points, periodic orbits) bifurcation theory is successful for obtaining solutions to the problems 1. - 4., see, e.g. the monographs Chow/Hale [3], Ruelle [18], and also Wiggins [20]. The book [16] by Palis/de Melo presents some basic concepts and results on structural stability.

Unfortunately, the program 1. - 4. turns out to be very difficult due to the following obstacles (among others): 
- The structure of the limit sets may be topologically quite complicated. In particular, they need not be isolated and the flow restricted to the limit sets need not be topologically nice (e.g. mixing) and need not define natural invariant measures.

- The Lyapunov spectrum is difficult to characterize and, in general, has no reasonable continuity properties.

For these reasons, one introduces some modified concepts, which we describe briefly. To simplify notations, we assume that the manifold $M$ is compact. A compact invariant set $A \subset M$ is an attractor, if it admits a neighborhood $N$ such that $A=\omega(N):=\left\{y \in M\right.$, there exist $t_{k} \uparrow \infty$ and $x_{k} \in N$ such that $\left.y=\lim _{k \rightarrow \infty} \Phi\left(t_{k}, x_{k}\right)\right\}$. Similarly, a repeller is a compact invariant set $R \subset M$ which has a neighborhood $N^{*}$ with $\omega^{*}\left(N^{*}\right)=R$. If $A \subset M$ is an attractor, then the set $A^{*}=\{y \in M, \omega(y) \cap A=\emptyset\}$ is its complementary repeller, and the pair $\left(A, A^{*}\right)$ is an attractor-repeller decomposition of $M$. In particular, if $x \notin A \cup A^{*}$, then $\omega^{*}(x) \subset A^{*}$ and $\omega(x) \subset A$. The set $\mathcal{R}=\bigcap\left\{A \cup A^{*}, A\right.$ is an attractor $\}$ is the chain recurrent set of the flow (1) and it contains all limit sets. If $\mathcal{R}$ has only finitely many components $\mathcal{M}_{1}, \ldots, \mathcal{M}_{n}$, then the $\mathcal{M}_{i}$ are the Morse sets (of the finest Morse decomposition) of the system, carrying an order which is induced by the relation $\mathcal{M}_{i} \prec \mathcal{M}_{j}$ iff there exists $x \in M$ with $\omega^{*}(x) \subset \mathcal{M}_{i}$ and $\omega(x) \subset \mathcal{M}_{j}$. The Morse sets and hence all attractors and repellers, are isolated invariant sets of the system, and for a continuous family $\Phi^{\alpha}, \alpha \in I \subset \mathbb{R}^{p}$, of dynamical systems the Morse sets depend upper semicontinuously on $\alpha$. Hence Morse decompositions are a suitable starting point for the study of dynamical systems using topological tools. But the flow (1) restricted to a Morse set is, in general, not topologically mixing or transitive. For the study of $\Phi$ on one of the $\mathcal{M}_{i}$ one needs the more general concept of chains: For $x, y \in M$ and $\varepsilon, T>0$ an $(\varepsilon, T)$-chain from $x$ to $y$ is given by a finite sequence of points $x_{0}=x, x_{1}, \ldots, x_{n}=y$ in $M$ and times $T_{0}, \ldots, T_{n-1} \geq T$ such that $d\left(\Phi\left(T_{i}, x_{i}\right), x_{i+1}\right)<\varepsilon$ for all $i$, where $d(\cdot, \cdot)$ denotes a metric on $M$. A subset $N \subset M$ is chain transitive, if for all $x, y \in N$ and all $\varepsilon, T>0$ there exists an $(\varepsilon, T)$-chain from $x$ to $y$. A point $x$ is chain recurrent if for all $\varepsilon, T>0$ there exists an $(\varepsilon, T)$-chain from $x$ to $x$. The set $\mathcal{R}$ defined above is exactly the set of all chain recurrent points of the flow. Furthermore, the flow $\Phi$ restricted to a Morse set $\mathcal{M}_{i}$ is chain transitive (and, of course, chain recurrent). We refer the reader to [8, Appendix B] for some basic facts on attractors, Morse decompositions, and chain recurrence, and to Conley [10], Ruelle [18], Robinson [17] for a detailed analysis of dynamical systems using these concepts.

A closer analysis of the asymptotic behavior of dynamical systems is possible via its linearization, the resulting stable, center, and unstable bundles, and the 
corresponding stable, center, and unstable manifolds. Briefly, the basic idea goes as follows: For a vector field $X \in \mathfrak{X}(M)$ denote by $T X \in T \mathfrak{X}(M)$ its linearization on the tangent bundle $T M$. The vector field $T X$ induces a flow on $T M$

$$
T \Phi: \mathbb{R} \times T M \longrightarrow T M
$$

which splits into its compact component on the projective bundle $\mathbb{P} M$

$$
\mathbb{P} \Phi: \mathbb{R} \times \mathbb{P} M \longrightarrow \mathbb{P} M
$$

and its linear component, the cocycle over $\mathbb{P} \Phi$

$$
D \Phi(t, x): T_{x} M \longrightarrow T_{\Phi(t, x)} M
$$

The compact component (3) on $\mathbb{P} M$ is independent of (4) and is studied via the concepts described above, while the linear component (4) determines the exponential convergence behavior via the Lyapunov exponents: For $x \in M$ and $v \in T_{x} M$ we define

$$
\lambda(x, v)=\lim \sup _{t \rightarrow \infty} \frac{1}{t} \log |D \Phi(t, x) v|
$$

If $J \subset M$ is an invariant set of (1), its Lyapunov spectrum is given by

$$
\Sigma_{L y}(J)=\left\{\lambda(x, v), x \in J, v \in T_{x} M\right\}
$$

For a fixed point $x^{*}$ of (1), i.e., $X\left(x^{*}\right)=0$, the set $J=\left\{x^{*}\right\}$ is invariant, the linearization of the vector field $X$ at $x^{*}$ is simply the Jacobian matrix $D_{x} X\left(x^{*}\right)=$ : $A$ and $\Sigma_{L y}\left(\left\{x^{*}\right\}\right)$ consists of the real parts of the eigenvalues of $A$. Similarly, for a periodic trajectory $\gamma \subset M$ of (1) the Lyapunov spectrum $\Sigma_{L y}(\gamma)$ consists of the Floquet exponents of the linearization over $\gamma$, which is a periodic matrix function. In both cases, the lim sup in (5) is actually a limit and the Lyapunov spectrum consists of finitely many numbers. The stable, center, and unstable subbundles of $T_{x^{*}} M$ (and of $T_{\gamma} M$, respectively) are easy to obtain by computing the eigenvectors of the linearization. These bundles are 'projected' onto $M$ to yield the stable, center, and unstable manifolds of the flow, locally at the fixed point $x^{*}$ (and at the periodic trajectory $\gamma$, respectively). But, in general, the cocycle (4) is a time varying matrix function in $T M$, whose Lyapunov spectrum and corresponding subbundle decomposition are difficult to obtain. Furthermore, for a family $\Phi^{\alpha}$ of dynamical systems the Lyapunov spectrum has, in general, no reasonable continuity properties in $\alpha$. Therefore, one often considers modified spectral concepts. One approach is to study the regular spectrum of $\Phi$ over 
an invariant set $J \subset M$, i.e. parts of the Lyapunov spectrum for which the exponents are given by limits and for which 'nice' subbundle decompositions exist. This is accomplished via the Multiplicativ Ergodic Theorem of Oseledets, which guarantees regularity of the spectrum with $\mu$-measure one for any $\Phi$-invariant measure $\mu$ in $J$, compare [15]. Another approach is to extend the Lyapunov spectrum to the topological spectrum, which contains all numbers $\lambda \in \mathbb{R}$ such that the flow $e^{\lambda t} \Phi(t, \cdot)$ does not admit an exponential dichotomy, compare Sacker/Sell [19]. The topological spectrum, which contains all Lyapunov exponents, enjoys an upper semicontinuity property for continuous families $\Phi^{\alpha}$ of flows, cp. Coppel [11], Johnson/Palmer/Sell [14].

Motivated by the concepts for the study of the flow $\Phi$ on $M$, one might also try to develop a spectral concept using attractor-repeller decompositions and Morse sets of $\Phi$ and of the compact component $\mathbb{P} \Phi$ of the linearization. This leads to the Morse spectrum, which we introduce in the next section, where we discuss generalizations of concepts from the theory of dynamical systems to control systems. These generalizations are a first step towards the study of the dynamic behavior of semigroups, because they lead from one-parameter semigroups (i.e., $\Phi(t, \cdot), t \in \mathbb{R})$ to more general semigroups of diffeomorphisms on a manifold, or to linear semigroups on flag manifolds.

\section{Control Theory}

The dynamic behavior of control systems is studied via an associated flow which leads to a semigroup of homeomorphisms (or diffeomorphisms) on the state space as explained in the next section. While dynamical systems, treated as in Section 2. generate one parameter groups of homeomorphisms on the state space $M$, a control system gives rise to two semigroups (forward or backward in time), but the basic concepts for dynamical and for control systems are analogous. We start with a brief description of control systems and their associated flows.

The prototype of a nonlinear control system is of the form

$$
\dot{x}=X_{0}(x)+\sum_{i=0}^{m} u_{i}(t) X_{i}(x)
$$

where we assume that the manifold $M$ and the vector fields $X_{0}, \ldots, X_{m}$ are smooth. The admissible control functions $u: \mathbb{R} \rightarrow U \subset \mathbb{R}^{m}$ may be measurable or piecewise constant, or of some regularity type between these two possibilities. For the following results we assume that $U$ is a compact, convex subset of $\mathbb{R}^{m}$ with 
$0 \in \operatorname{int} U$. We denote by

$$
\mathcal{U}=\{u: \mathbb{R} \rightarrow U, \text { measurable }\}
$$

the set of admissible control functions.

Associated with the control system (7), (8) is the so-called control flow

$$
\Phi: \mathbb{R} \times \mathcal{U} \times M \longrightarrow \mathcal{U} \times M,(t, u, x) \longmapsto\left(\Theta_{t} u, \varphi(t, x, u)\right)
$$

Here $\theta: \mathbb{R} \times \mathcal{U} \longrightarrow \mathcal{U},(t, u) \longmapsto u(t+\cdot)$ is the shift on $\mathcal{U}$, and $\varphi(t, x, u)$ denotes the solution of $(7)$ at time $t$ under the control function $u \in \mathcal{U}$ with $\varphi(0, x, u)=x$ as initial value. If $\mathcal{U} \subset L_{\infty}(\mathbb{R}, \mathbb{R})$ is endowed with the weak* topology, then $\Phi$ becomes a continuous flow of homeomorphisms on $\mathcal{U} \times M$, which can be analyzed using topological dynamics.

Just as for dynamical systems in Section 2., one can use (controlled) trajectories or (controlled) chains for the study of the dynamic behavior. The trajectory point of view leads to control sets of (7) and to transitivity of (9), while chains give rise to chain control sets of (7) and to chain transitivity of (9).

Controllability and Transitivity

For the control system (7) we define the positive orbit $\mathcal{O}^{+}(x)$ and the negative orbit $\mathcal{O}^{-}(x)$ for a point $x \in M$ as

$$
\begin{aligned}
& \mathcal{O}^{+}(x)=\{y \in M, \text { there exist } u \in \mathcal{U} \text { and } t \geq 0 \text { with } y=\varphi(t, x, u)\} \\
& \mathcal{O}^{-}(x)=\{y \in M, \text { there exist } u \in \mathcal{U} \text { and } t \geq 0 \text { with } x=\varphi(t, y, u)\}
\end{aligned}
$$

A set $D \subset M$ with nonvoid interior is a control set if $D$ is a maximal set with the property that $D \subset \operatorname{cl} \mathcal{O}^{+}(x)$ for all $x \in D$.

We impose a condition that guarantees int $O^{ \pm}(x) \neq \emptyset$ for all $x \in M$ :

$$
\operatorname{dim} \mathcal{L} \mathcal{A}\left\{X_{0}+\sum_{i=1}^{m} u_{i} X_{i}, u \in U\right\}(x)=\operatorname{dim} M \text { for all } x \in M
$$

The main result on the connection of control sets for (7) and transitivity for (9) can be formulated as (compare [5]):

Theorem 1 Consider the control system (7) and assume that (H) holds. For a control set $D \subset M$ define the lift to $\mathcal{U} \times M$ as

$$
\mathcal{D}=\operatorname{cl}\{(u, x) \in \mathcal{U} \times M, \varphi(t, x, u) \in \operatorname{int} D \text { for all } t \in \mathbb{R}\}
$$

Then $\mathcal{D}$ is a maximal topologically transitive and topologically mixing component of $(\mathcal{U} \times M, \Phi)$. Vice versa, if $\mathcal{D} \subset \mathcal{U} \times M$ is maximal topologically mixing with $\operatorname{int} \pi_{M} \mathcal{D} \neq \emptyset$, then there exists a control set $D \subset M$ such that $\mathcal{D}$ is its lift. 
There is a reachability order $\preceq$ between control sets given by

$$
D \preceq D^{\prime} \text { if there exists } x \in D \text { with } \operatorname{cl}^{+}(x) \cap D^{\prime} \neq \emptyset
$$

This order describes the global reachability structure of (7) and for compact $M$ the invariant control sets $C$ (i.e. $C=\operatorname{cl}^{+}(x)$ for all $x \in C$ ) are maximal with respect to this order.

Control sets need not contain all the limit sets of a control system, and they need not be isolated. (These remarks also remain true, if slightly a slightly more general notion of control sets is used which allows for void interior.) As in the case of dynamical systems, one therefore introduces the notion of controlled chains.

\section{Chain Controllability and Chain Transitivity}

A controlled $(\varepsilon, T)$-chain from $x$ to $y$ is given by finite sequences of points $x_{0}=x, \ldots, x_{n}=y$ in $M$, of controls $u_{0}, \ldots, u_{n}$ in $\mathcal{U}$, and of times $T_{0}, \ldots, T_{n} \geq T$ such that $d\left(\varphi\left(T_{i}, x_{i}, u_{i}\right), x_{i+1}\right)<\varepsilon$ for $i=0,1, \ldots, n-1$. The positive chain orbit $\mathcal{O}_{c}^{+}(x)$ is given as

$$
\mathcal{O}_{c}^{+}(x)=\left\{y \in M, \quad \begin{array}{l}
\text { for all } \varepsilon, T>0 \text { there exists a controlled } \\
(\varepsilon, T) \text {-chain from } x \text { to } y
\end{array}\right\}
$$

Chain control sets of (7) are now defined similar to control sets with $\mathcal{O}^{+}(x)$ replaced by $\mathcal{O}_{c}^{+}(x)$ : a chain control set $E \subset M$ is a maximal set with the property that $E \subset \operatorname{cl} \mathcal{O}_{c}^{+}(x)$ for all $x \in E$ and for all $x \in E$ there is a control $u \in \mathcal{U}$ with $\varphi(t, x, u) \in E$ for all $t \in \mathbb{R}$. We emphasize that the definition of chain orbits and hence of chain control sets contains the concept of time via the requirement $T_{i} \geq T$ for all $T>0$, while positive orbits and control sets do not depend explicitly on an idea of time.

The analogue of Theorem 1 for chains takes the following form.

Theorem 2 Consider the control system (7) and let $E \subset M$ be a chain control set with lift

$$
\mathcal{E}=\{(u, x) \in \mathcal{U} \times M, \varphi(t, x, u) \in E \text { for all } t \in \mathbb{R}\}
$$

Then $\mathcal{E}$ is a maximal chain transitive set of $(\mathcal{U} \times M, \Phi)$. Vice versa, let $\mathcal{E} \subset \mathcal{U} \times M$ be a maximal chain transitive set of (9), then $\pi_{M} \mathcal{E} \subset M$ is a chain control set of (7).

The proof of this result can be found in [5]. If $M$ is compact, then $\mathcal{U} \times$ $M$ is a compact space and therefore the chain recurrent components of $(\mathcal{U} \times$ 
$M, \Phi)$ are the maximal chain transitive sets, which define a unique finest Morse decomposition of the control flow (9). The associated order induces an order of the chain control sets of $(7)$ on $M$, because the shift space $(\mathcal{U}, \Theta)$ is chain recurrent. Hence one obtains a global structure of the control system with respect to chain controllability. This order and the reachability order between the control sets agree, if all chain control sets of (7) are closures of control sets. The following discussion shows, under which conditions one can expect an agreement.

\section{Controllability and Chain Controllability}

There are different ways of imbedding the control system (7) into a family of such systems. Here we introduce one such imbedding, which has a natural extension to semigroups, compare Section 4. At the same time, this imbedding reveals relations between trajectories and chains of the system (7), and of various spectral concepts, as described below.

The basic idea is to vary the control range $U$ of the admissible control functions. For $\rho \geq 0$ we denote

$$
U^{\rho}=\rho \cdot U, \mathcal{U}^{\rho}=\left\{u: \mathbb{R} \rightarrow U^{\rho} \text {, measurable }\right\}
$$

leading to a family $(7)^{\rho}$ of control systems and $\Phi^{\rho}$ of control flows. We need an inner pair condition which guarantees that larger control ranges increase the corresponding system orbits:

$$
\begin{aligned}
\text { For } 0 & \leq \rho<\rho^{\prime}<\infty \text { we require that for all }(u, x) \in \mathcal{E}^{\rho} \\
\text { there exists } t & >0 \text { such that } \varphi(t, x, u) \in \operatorname{int} \mathcal{O}^{+, \rho^{\prime}}(x)
\end{aligned}
$$

Here $\mathcal{E}^{\rho}$ are the lifts of the chain control sets of $(7)^{\rho}$ and $\mathcal{O}^{+, \rho^{\prime}}(x)$ are the positive orbits from $x$ of the control system $(7)^{\rho^{\prime}}$.

We obtain the following result (see [6], [8]).

Theorem 3 Consider the family ( $\left.{ }^{\prime}\right)^{\rho}, \rho \geq 0$, of control systems under the Assumptions ( $H)$ and (I), and let $M$ be compact.

(i) For each $\rho^{*}>0$ and each chain control set $E^{\rho^{*}}$ of (7) $)^{\rho^{*}}$ there exist control sets $D^{\rho}$ for $\rho>\rho^{*}$ such that $E^{\rho^{*}}=\bigcap_{\rho>\rho^{*}} D^{\rho}$. (For $\rho^{*}=0$ the $E^{\rho^{*}}$ is any of the Morse sets of the uncontrolled system $\dot{x}=X_{0}(x)$.)

(ii) For all but at most countably many $\rho>0$ we have that for all chain control sets $E^{\rho}$ there exists a control set $D^{\rho}$ such that $E^{\rho}=\operatorname{cl} D^{\rho}$. In particular, if $\omega(u, x)$ (or $\left.\omega^{*}(u, x)\right)$ is a limit set of $\Phi^{\rho}$, then $\pi_{M} \omega(u, x)$ (and $\pi_{M} \omega^{*}(u, x)$ ) is contained in the closure of some control set of $(7)^{\rho}$.

(iii) The control sets $D^{\rho}$ and the chain control sets $E^{\rho}$ depend continuously on $\rho$ (in the Hausdorff topology) for all $\rho$ where (i) holds. 
Theorem 3 is the starting point of a bifurcation theory for control systems with varying control range: The 'pathological' systems of the family $(7)^{\rho}$ are those systems for which there exists a chain control set which is not the closure of a control set. For these exceptional $\rho$ 's the (chain) control sets vary discontinuously, and the closures of the control sets may not contain all the limit points of the system. On the other hand, we can describe for all $\rho^{*} \geq 0$ the chain control sets $E^{\rho^{*}}$ as limits of control sets $D^{\rho}$ for $\rho \downarrow \rho^{*}$ according to Theorem 3(i). Hence we have found, under Assumption (I), a characterization of the chain control sets that does not depend explicitly on a concept of time and which, therefore, allows for a generalization to certain abstract semigroups, compare Section 4. This range of ideas can be made more precise for bilinear control systems, for which the vector fields $X_{0}, \ldots, X_{m}$ are linear, compare [4] and Section 4.

\section{The Linearized Control System}

A more detailed study of the dynamic behavior of control systems is possible via the linearized system - just as for dynamical systems. We briefly explain some of the basic ideas of linearization, its compact and radial component, and Lyapunov exponents. For the details on these concepts and for associated stable manifolds we refer to [8].

Let $X$ be a smooth vector field on a smooth manifold $M$. We denote by $T X$ the linearized vector field on $T M$, the tangent bundle of $M$. With this notation the linearization of the control system (7) takes the form

$$
(T x)^{\cdot}=T X_{0}(T x)+\sum_{i=1}^{m} u_{i}(t) T X_{i}(T x) \quad \text { on } T M
$$

where $T x \in T M$. Writing $T X=(X, D X)$ the corresponding flow reads with $v \in T_{x} M$

$$
T \Phi: \mathbb{R} \times \mathcal{U} \times T M \rightarrow \mathcal{U} \times T M,(t, u, x, v) \longmapsto\left(\Theta_{t} u, D \varphi(t, x, u) v\right)
$$

As in (2)-(4) this flow splits into a compact component on $\mathbb{P} M$

$$
\mathbb{P} \Phi: \mathbb{R} \times \mathcal{U} \times \mathbb{P} M \rightarrow \mathcal{U} \times \mathbb{P} M
$$

and a radial component, the cocycle over $\mathbb{P} \Phi$

$$
D \varphi(t, x, u): T_{x} M \rightarrow T_{\varphi(t, x, u)} M
$$

Note that the cocycle (16) depends on all three components $(t, u, x) \in \mathbb{R} \times \mathcal{U} \times M$. The dynamic behavior of the compact component (15), which is a control flow 
itself, is studied via the theory outlined above, using the Assumptions (H) and (I) for the control system on the projective bundle. If $M$ is compact, then $\mathbb{P} M$ is a compact space, and hence Theorems $1-3$ are valid with the obvious modifications. It remains to study the radial component (16), which we will do for the special case of a control system with singular point. The general theory can be found in $[8]$.

Consider the control system (7) and let $x^{*} \in M$ be a singular point, i.e., $X_{0}\left(x^{*}\right)=\ldots=X_{m}\left(x^{*}\right)=0$. Since $\varphi\left(t, x^{*}, u\right)=x^{*}$ for all $t \in \mathbb{R}, u \in U$, the linearization at $x^{*}$ can be identified with the bilinear control system

$$
\dot{v}=A_{0} v+\sum_{i=1}^{m} u_{i}(t) A_{i} v \quad \text { in } \mathbb{R}^{d}(d=\operatorname{dim} M)
$$

where $A_{i}=D_{x} X_{i}\left(x^{*}\right)$ is the Jacobian of $x^{*}$ at $x^{*}$ for $i=0, \ldots, m$. The compact component of (17) reads

$$
\dot{p}=h(p, u)=h_{0}(p)+\sum_{i=1}^{m} u_{i}(t) h_{i}(p) \quad \text { in } \mathbb{P}^{d-1}
$$

with $h_{i}(p)=\left(A_{i}-q_{i}(p, u) \cdot I d\right) p, q_{i}(p, u)=p^{T} A_{i} p$. Note that (18) is a (real analytic) system of the form (7), hence Theorems 1-3 describe its dynamic behavior. The cocycle over (18) is for $t \in \mathbb{R}, u \in \mathcal{U}$, and any $v \in \mathbb{R}^{d} \backslash\{0\}$ which projects to $p \in \mathbb{P}^{d-1}$ given by

$$
\sigma(t, u, p)=\frac{|\psi(t, v, u)|}{|v|}
$$

where $\psi(t, x, u)$ denotes the solution of $(17)$ and $|\cdot|$ is a prescribed norm in $\mathbb{R}^{d}$. An alternative description is given by

$$
\sigma(t, u, p)=\exp \int_{0}^{t} q(p, u) d \tau
$$

where $q(p, u)=q_{0}(p)+\sum_{i=1}^{m} u_{i}(\tau) q_{i}(p)$. Its dynamic behavior is described by the Lyapunov exponents and the associated Lyapunov subspaces of $\mathcal{U} \times \mathbb{R}^{d}$ :

$$
\lambda(u, v)=\lim \sup _{t \rightarrow \infty} \frac{1}{t} \log |\psi(t, v, u)|
$$

We denote the Lyapunov spectrum of (17) by

$$
\Sigma_{L y}=\left\{\lambda(u, v),(u, v) \in \mathcal{U} \times \mathbb{R}^{d}, v \neq 0\right\}
$$


As for dynamical systems the spectrum $\Sigma_{L y}$ cannot be characterized easily. Therefore, one rewrites the exponents in terms of the solutions of (18)

$$
\lambda(u, v)=\lim \sup _{t \rightarrow \infty} \frac{1}{t} \int_{0}^{t} q(p, u) d \tau
$$

and uses the control and chain control structure of the control system (18) to obtain inner and outer approximations of the Lyapunov spectrum.

\section{The Lyapunov Spectrum}

The control system (18) has a finite number of control sets $D_{1}, \ldots, D_{k}, k \leq d$, which are linearly ordered through the reachability order (11). Without loss of generality we set $D_{1} \preceq \ldots \preceq D_{k}$. We define the Floquet spectrum of each $D_{i}$ as

$$
\Sigma_{F l}\left(D_{i}\right)=\left\{\lambda(u, v), \begin{array}{l}
u \text { is piecewise constant periodic with } \\
\text { period } T \text { and } \psi(T, v, u)=v \in \operatorname{int} D_{i}
\end{array}\right\}
$$

The closures $\mathrm{cl} \Sigma_{F l}\left(D_{i}\right)$ are intervals which are ordered in the same way as the control sets, i.e., inf $\operatorname{cl} \Sigma_{F l}\left(D_{i}\right) \leq \inf \operatorname{cl} \Sigma_{F l}\left(D_{j}\right)$ and $\operatorname{supcl} \Sigma_{F l}\left(D_{i}\right) \leq \sup \operatorname{cl} \Sigma_{F l}\left(D_{j}\right)$ for $i<j$. However, the intervals may overlap. It holds that $\Sigma_{L y} \supset \bigcup_{i=1}^{k} \operatorname{cl} \Sigma_{F l}\left(D_{j}\right)$.

On the other hand, the control system (18) has $l$ chain control sets $(1 \leq l \leq$ $k \leq d) E_{1} \preceq \ldots \preceq E_{l}$, which are ordered by the Morse decomposition structure of the control flow associated with (18). We define the Morse spectrum of each $E_{j}$ in the following way:

Let $\zeta=\left(x_{r}, u_{r}, T_{r}, r=0, \ldots, n\right)$ be a controlled $(\varepsilon, T)$-chain in $E_{j}$, denote by

$$
\lambda(\zeta)=\frac{1}{\sum_{i=0}^{n-1}} \log \frac{|\psi(T, v, u)|}{|v|}=\frac{1}{\sum_{i=0}^{n-1}} \sum_{i=1}^{n} \int_{0}^{T_{i}} q(p, u) d \tau
$$

the (finite time) exponent of $\zeta$, and define

$$
\Sigma_{M o}\left(E_{j}\right)=\left\{\lambda \in \mathbb{R}, \begin{array}{l}
\text { there exist sequences } \varepsilon_{\alpha} \downarrow 0 \text { and } T_{\alpha} \uparrow \infty \text { and } \\
\left(\varepsilon_{\alpha}, T_{\alpha}\right) \text {-chains } \zeta_{\alpha} \text { with } \lambda=\lim _{\alpha \rightarrow \infty} \lambda\left(\zeta_{\alpha}\right)
\end{array}\right\}
$$

The sets $\Sigma_{M o}\left(E_{j}\right)$ are intervals, which are ordered in the same way as the chain control sets. Hence the order of the Morse spectral intervals reflects the attractorrepeller decompositions of the control flow associated to the system (18). Each chain control set $E_{j}$ contains at least one control set and we have

$$
\begin{aligned}
\Sigma_{M o}\left(E_{j}\right) & \supset \Sigma_{L y}\left(E_{j}\right)=\left\{\lambda(u, v), \psi(t, v, u) \subset E_{j} \text { for all } t \geq 0\right\} \\
& \supset \bigcup \operatorname{cl} \Sigma_{F l}(D), D \subset E_{j} \text { is a control set } \\
\bigcup_{j=1}^{l} \Sigma_{M o}\left(E_{j}\right) & \supset \Sigma_{L y} \supset \bigcup_{i=1}^{k} \operatorname{cl} \Sigma_{F l}\left(D_{i}\right)
\end{aligned}
$$


Note that all three spectral concepts require an idea of time according to the definitions (20), (22), and (23), although the requirement of periodicity in the definition (22) of the Floquet spectrum is only related to the positive orbit of the control system (18).

The relation between the different spectral concepts in (24) can again be studied via imbedding the control systems (17) and (18) into a family of systems with varying control range as in (13) (see [8]).

Theorem 4 Consider the family $(18)^{\rho}, \rho \geq 0$, of control systems under Assumptions $(H)$ and $(I)$.

(i) For all but at most countably many $\rho>0$ we have that

$$
\Sigma_{M o}\left(E_{j}^{\rho}\right)=\Sigma_{L y}\left(E_{j}^{\rho}\right)=\operatorname{cl} \Sigma_{F l}\left(D_{j}^{\rho}\right), j=1, \ldots, k(\rho)
$$

where $D_{j}^{\rho}$ is the unique control set with $\mathrm{cl} D_{j}^{\rho}=E_{j}^{\rho}$ (compare Theorem 3).

(ii) The spectral intervals depend continuously on $\rho$ for all $\rho$ where (i) holds.

At the exceptional $\rho$-values from Theorem 4(i) we have the following situation: There exists a pair $(u, v) \in \mathcal{U} \times \mathbb{R}^{d}, v \neq 0$, and a chain control set $E \subset \mathbb{P}^{d-1}$ such that $\pi_{\mathbb{P}^{d-1}} \omega(u, v) \subset E \backslash(\bigcup$ int $D)$, where the union is taken over all control sets contained in $E$. This can occur, in particular, at the discontinuity points of the (chain) control sets of the system (18), compare Theorem 3. Here the limit structure of the control system on the compact component $\mathbb{P}^{d-1}$ enters into the spectral theory, i.e., the exponential behavior of the radial component.

Associated with the Morse spectrum is a decomposition of the bundle $\mathcal{U} \times \mathbb{R}^{d}$ into Morse subspaces

$$
\left.\mathcal{U} \times \mathbb{R}^{d}=\bigoplus_{j=1}^{l} \mathcal{V}_{j} \quad \text { (Whitney sum }\right)
$$

such that $\pi_{\mathbb{P}^{d-1}} \mathcal{V}_{j} \subset E_{j}$ and $(u, v) \in \mathcal{V}_{j}$ implies $\lambda(u, v) \in \Sigma_{M o}\left(E_{j}\right)$. This decomposition is continuous in $u \in \mathcal{U}$, i.e. the $\mathcal{V}_{j}$ are constant dimensional in $u \in \mathcal{U}$. This fact may open the door for a topological classification of bilinear control systems, compare Section 6.

Similar to the situation for dynamical systems the subbundles $\mathcal{V}_{j}$ 'project' onto invariant manifolds of the original nonlinear system locally at the singular point $x^{*}$, compare [8]. However, we will not consider implications of this fact in the present paper. 


\section{Semigroups and Control Theory}

In the study of a control system Lie semigroups enter through the so called systems semigroup. Starting with the system (7) suppose that its control functions are taken within the set piecewise constant functions. Then the trajectories are given by concatenations of trajectories of the family of vector fields

$$
N=\left\{X_{0}+\sum_{i=1}^{m} u_{i} X_{i}, u \in U\right\}
$$

Hence if a trajectory starts at $x$ and ends at $y \in M$, we have that $y$ is the image of $x$ under successive compositions of flows of vector fields of $N$. These compositions give rise to the 'semigroup'

$$
S=\left\{Y_{t_{1}}^{1} \circ \cdots \circ Y_{t_{k}}^{k}: t_{i} \geq 0, Y^{i} \in N\right\}
$$

Here $Y_{t}$ stands for the flow of the vector field $Y$. The systems semigroup is naturally imbedded in the systems group

$$
G=\left\{Y_{t_{1}}^{1} \circ \cdots \circ Y_{t_{k}}^{k}, t_{i} \in \mathbb{R}, Y^{i} \in N\right\}
$$

In general $S$ and $G$ are huge sets of local diffeomorphisms of $M$. However if one knows beforehand that the vector fields in $N$ come from the action a Lie group, say $H$, then $G$ turns out to be a Lie subgroup of $H$ and $S$ a subsemigroup of $G$. More precisely, suppose that $\phi: H \times M \rightarrow M$ is an action of the Lie group $H$ on $M$. If $\mathfrak{h}$ stands for the Lie algebra of $H$ then each $Y \in \mathfrak{h}$ induces the vector field

$$
\tilde{Y}(x)=\frac{d}{d t} \phi(\exp t Y, x)_{\mid t=0}
$$

on $M$. The flow of $\tilde{Y}$ is just the action of $\exp (t Y)$. Hence if the vector fields in $N$ are of the form $X_{i}=\tilde{Y}_{i}$ with $Y_{i} \in \mathfrak{h}, i=0, \ldots, m$, we have that $G$ is a subgroup of $H$. Moreover, $G$ is a nice connected submanifold of $H$ which makes it a Lie subgroup. Its Lie algebra $\mathfrak{g}$ is the subalgebra of $\mathfrak{h}$ generated by $\left\{Y_{0}, \ldots, Y_{m}\right\}$. This can be seen by considering the system lifted to the Lie group:

$$
\dot{g}=Y_{0}^{*}(g)+\sum_{i=1}^{m} Y_{i}^{*}(g), \quad g \in H
$$

Here $Y^{*}$ stands for the right invariant vector field in $H$ defined by $Y \in \mathfrak{h}$. The trajectories of the original system are "projections" of the trajectories of (25), in 
the sense that $\phi(\alpha(t), x)$ is a trajectory starting at $x \in M$ if $\alpha$ is a trajectory of (25), and every trajectory of the system in $M$ is obtained this way. The systems group $G$ turns out to be the orbit of (25), that is, the set of points reachable form the identity in $H$, forward and backwards in time. In the same way, $S$ is the forward orbit of the identity in $H$. Since $\mathfrak{g}$ is generated by $\left\{Y_{0}, \ldots, Y_{m}\right\}$ it follows that (25) satisfies the Lie algebra rank condition $(\mathrm{H})$. This condition implies in particular that $S$ has nonempty interior in $G$, and moreover

$$
\operatorname{clint} S=\operatorname{cl} S .
$$

One of the main classes of systems on Lie groups are the bilinear systems (17) discussed in Section 3 : For $d \times d$ matrices $A_{i}, i=0, \ldots, m$ let

$$
\dot{x}=A_{0} x+\sum_{i=1}^{m} u_{i}(t) A_{i} x
$$

be the system in $\mathbb{R}^{d}$ with $u \in \mathcal{U}$. Such a system can be lifted to the system

$$
\dot{g}=A_{0} g+\sum_{i=1}^{m} u_{i}(t) A_{i} g, \quad g \in \mathrm{Gl}(d, \mathbb{R})
$$

in the general linear group $\mathrm{Gl}(d, \mathbb{R})$. The solutions of $(26)$ are the fundamental solutions of (17). The systems group $G$ is a connected Lie subgroup of $\operatorname{Gl}(d, \mathbb{R})$ and the systems semigroup $S$ has nonempty interior in $G$.

For the asymptotic analysis of (17) we use the polar decomposition of $\mathbb{R}^{d}$. Let $\mathbb{P}^{d-1}$ be the projective space and $\pi: \mathbb{R}^{d} \backslash\{0\} \rightarrow \mathbb{P}^{d-1}$ the canonical projection. This projection turns $\mathbb{R}^{d} \backslash\{0\}$ into a fiber bundle over $\mathbb{P}^{d-1}$ (actually a principal bundle with $\mathbb{R} \backslash\{0\}$ as structure group). The system (26) induces a system

$$
\dot{s}=\tilde{A}_{0}(s)+\sum_{i=1}^{m} u_{i}(t) \tilde{A}_{i}(s), \quad s \in \mathbb{P}^{d-1}
$$

on $\mathbb{P}^{d-1}$, which is the projection of (17) onto $\mathbb{P}^{d-1}$ and is also the system induced by (26) through the transitive action of $\mathrm{Gl}(d, \mathbb{R})$ on $\mathbb{P}^{d-1}$. Complementary to (27) is the radial component of (17). This component is described by a cocycle of the $\mathrm{Gl}(d, \mathbb{R})$-action on $\mathbb{P}^{d-1}$ as follows: For $g \in \mathrm{Gl}(d, \mathbb{R})$ and $s \in \mathbb{P}^{d-1}$ put

$$
\sigma(g, s)=\frac{|g x|}{|x|}
$$


where $x$ is any representative of $s$ in $\mathbb{R}^{d} \backslash\{0\}$, and $|\cdot|$ is a prescribed norm in $\mathbb{R}^{d}$. Then $\rho$ satisfies the cocycle property

$$
\sigma(g h, s)=\sigma(g, h s) \sigma(h, s) .
$$

The bilinear system (17) decomposes into the compact component (27) and the cocycle (28) so that the asymptotic behavior of (17) is described by the dynamical properties of (27) together with the behavior of (28) at $\infty$.

A similar polar decomposition occurs on the level of $\operatorname{Gl}(d, \mathbb{R})$ on the flag manifolds. In order to explain this decomposition we let $\mathbb{F}\left(r_{1}, \ldots, r_{s}\right)$ be a flag manifold. Its elements are flags of the type

$$
\left(V_{1} \subset \cdots \subset V_{s}\right)
$$

with $V_{i}$ a subspace of $\mathbb{R}^{d}$ of dimension $r_{i}$. In particular, we consider the full flag manifold $\mathbb{B}=\mathbb{F}(1,2, \ldots, d-1)$. The group $\operatorname{Gl}(d, \mathbb{R})$ acts transitively on any flag manifold. The action is given by the formula

$$
g\left(V_{1} \subset \cdots \subset V_{s}\right)=\left(g V_{1} \subset \cdots \subset g V_{s}\right)
$$

with $g$ an invertible matrix. Hence the bilinear system (26) on the group level induces systems on the flag manifolds. These systems form the compact part of (26) and they encompass the system on projective space. Note that the system on the full flag $\mathbb{B}$ projects onto the system on any other flag manifold and hence the controllability properties of the systems on the flag manifolds are embodied in those of the system on $\mathbb{B}$. The complementing radial part of the systems on the flag manifolds are the values of the cocycles in the trajectories. In view of the situation in projective space it appears natural to take cocycles for the $\operatorname{Gl}(d, \mathbb{R})$ action on $\mathbb{B}$ which are invariant under the orthogonal group in the sense that $\sigma(k, b)=1$ for all orthogonal matrices $k$ and all $b \in \mathbb{B}$.

The same kind of decomposition works for systems on a semi-simple Lie group. Let $G$ be such a group and take an Iwasawa decomposition $G=K A N$ of $G$. If $M$ denotes the centralizer of $A$ in $K$ then $P=M A N$ is a (minimal) parabolic subgroup and $B=G / P$ is a compact homogeneous space of $G$ and plays the role of the maximal flag manifold so that the compact component of a system on $G$ is described by the induced system on $B$. For the radial part we consider $K$-invariant cocycles for the $G$-action on $B$. These cocycle have a well known description (see e.g. ): For $g \in G$ write $g=K(g) A(g) N(g)$ for its Iwasawa decomposition. Let $\mathfrak{a}$ denote the Lie algebra of $A$ and $\mathfrak{a}^{*}$ its dual. Then any $K$-invariant cocycle on $B$ is of the form

$$
\sigma_{\lambda}(g, b)=e^{\lambda(\log A(g k))}
$$


where $\lambda \in \mathfrak{a}^{*}$ and $k \in K$ is such that $k b_{0}=b$ where $b_{0}=P$ is the origin of $B$. These cocycles have a geometric interpretation analogous to the decomposition $\mathbb{R}^{d} \backslash\{0\} \rightarrow \mathbb{P}^{d-1}$ which gives rise to the cocycle (28). In fact, consider the closed subgroup $M N$ and form the homogenous space $G / M N$. There is a canonical fibration

$$
G / M N \longrightarrow G / P
$$

Since $M N$ is normal in $P$ this is a principal bundle with structure group $A=$ $P / M N$. Incidentally this bundle is trivial, i.e., is diffeomorphic to $B \times A$ and it turns out that if $h$ denotes the $A$-component of $g(b, 1)$ in $B \times A$ then $\rho_{\lambda}(g, b)=$ $\exp (\lambda(\log h))$. This means that the $K$-invariant cocycles on $B$ are read off from the $A$-component of the decomposition of $G / M N$ and this component may be viewed as the radial part of the systems on $G$.

For the general nonlinear system (7) we consider the polar decomposition of its linearization: Let $B M$ be the frame bundle of $M$, which is a principal bundle with $\operatorname{Gl}(d, \mathbb{R})$ as structure group. For a vector field $X$ on $M$ we denote by $\tilde{X}$ its

lifting to $B M$. The flow $\tilde{X}_{t}$ of $\tilde{X}$ is given by the differential $d X_{t}$ of the flow of $X$. Let

$$
\dot{p}=\tilde{X}_{0}(p)+\sum_{i=0}^{m} u_{i}(t) \tilde{X}(p) \quad p \in B M
$$

be the lifting of (7) to $B M$. This system plays a role analogous to a system in a Lie group in the sense that it induces control systems on the fiber bundles associated to it, like e.g. the tangent bundle $T M$, the projective bundle $\mathbb{P} M$ or a flag bundle $\mathbb{F} M$ made of flags of $T M$. As for the bilinear systems we here have polar decompositions, which are obtained fiberwise from the linear decompositions explained above. For instance, the compact part of the system in $T M$ is the system induced on $\mathbb{P} M$, and its radial part is given by a norm as in (28).

\section{Asymptotic Behavior of Semigroups - the Compact Component}

In this section we outline the contours of a research program on the behavior of semigroups at $\infty$, and relate it to current research activities in semigroup theory, control theory, and the theory of dynamical systems. We will restrict our attention to subsemigroups of Lie groups with their specific structure, but we will try to keep topological and algebraic questions separate, whenever possible.

The first problem is basic for understanding any equivalence between semigroups and control systems: 
Problem 5.1 Given a connected Lie group $G$ characterize the systems (25) that generate the same semigroup $S$ (or $\operatorname{cl} S$ in $G$ ).

This seems to be a formidable problem and should be kept as a general goal. In fact, a particular instance of it is the controllability property in $G$, which is the problem of deciding whether $S=G$. This problem has been dealt extensively in the literature since the early seventies, and up to now has only partial solutions (compare e.g. Kupka....

Turning to the semigroups themselves one could try to characterize them through their transitivity and chain transitivity behavior. In order to discuss this question we restrict ourselves to linear semigroups acting on flag manifolds. This leads to the following problem.

Problem 5.2 Characterize the linear semigroups $S$ which have the same transitivity and/or chain transitivity behavior on the complete flag $\mathbb{B}=\mathbb{F}(1,2, \ldots, d-1)$.

As a motivation, recall that the flag manifold $\mathbb{B}$ contains the main geometric information about the group $\mathrm{Sl}(d, \mathbb{R})$. Hence one may expect that the same kind of information is encoded in this flag manifold at least for the semigroups $S$ in $\mathrm{Sl}(d, \mathbb{R})$ with nonempty interior. We know (c.f. ?)

1. Let $W$ be the permutation group in $d$ letters (i.e., the Weyl group of $\operatorname{Sl}(d, \mathbb{R}))$. Then there is a map $w \mapsto D_{w}$ that maps $W$ onto the set of control sets in the full flag $\mathbb{B}=\mathbb{F}(1,2, \ldots, d-1)$.

2. There is just one invariant control set $C$ which corresponds to the identity permutation. Let

$$
W(S)=\left\{w \in W: D_{w}=C\right\}
$$

Then $W(S)$ is a subgroup of $W$ and the number of control sets in $\mathbb{B}$ is $|W| /|W(S)|$.

3. The subgroup $W(S)$ can be recovered from $C$. In fact, there are integers $1 \leq r_{1} \leq \cdots \leq r_{s}<d$ such that $W(S)$ is the direct product

$$
\Pi\left[1, r_{1}\right] \times \cdots \times \Pi\left[r_{s}+1, d\right]
$$

where $\Pi[a, b]$ means the group of permutations of the numbers between $a$ and $b$. Put $\mathbb{F}^{\prime}=\mathbb{F}\left(r_{1}, r_{1}+r_{2}, \ldots, r_{1}+\cdots+r_{s}\right)$, and denote by $\pi: \mathbb{B} \rightarrow \mathbb{F}^{\prime}$ the canonical projection from the full flag. Then $C$ is the inverse image under $\pi$ of the unique invariant control set for the induced system on $\mathbb{F}^{\prime}$. 
The subgroup $W(S)$ is an invariant of the transitivity structure of $S$ on the flag manifold. Also note that the last statement above ensure that $W(S)$ can be recovered from the invariant control set in $\mathbb{F}$. In particular, two semigroups having the same invariant control set in $\mathbb{F}$ have also the same structure of control sets. This holds although the control sets themselves - and hence the transitivity structure - may be different. Consider for example the compression semigroup: Let $C \subset \mathbb{F}$ be the invariant control set of the semigroup $S$ and put

$$
\operatorname{Com}(C)=\{g \in G, g C \subset C\}
$$

Clearly $S \subset \operatorname{Com}(C)$ and the invariant control set of $\operatorname{Com}(C)$ is $C$. Hence there are as many control sets of $S$ in $\mathbb{B}$ as there are for $\operatorname{Com}(C)$, and any $S$-control set is contained in exactly one $\operatorname{Com}(C)$-control set. However, the control sets for $\operatorname{Com}(C)$ may be bigger than those for $S$. This suggests the following problem.

Problem 5.3 Given the control sets of $S$ in $\mathbb{F}(1,2, \ldots, d-1)$, construct and describe the maximal semigroup $S\left\{D_{w}, w \in W\right\}$ which has the same control sets as $S$.

These problems were stated with the assumption that $S$ has nonempty interior in $\mathrm{Sl}(d, \mathbb{R})$. As the theory outlined above extends to semi-simple Lie groups, the same questions should be posed for semigroups with interior points in a group $G$ in this class. Now, the Furstenberg boundaries of $G$ play the role of the above flag manifolds.

The same program needs to be carried out for the notion of chain control sets for semigroups as developed in [2]. Note that contrary to control sets the concept of chain control sets for a control system requires the evolution of time (compare Section 3.), so that the jumps are allowed only after the system runs for some time $T>T_{0}$. In a similar way the notion of chain control sets for semigroups requires the introduction of another object besides the semigroup. In [2] this new object was taken to be a family of subsets of $S$. In order to explain this construction we take the family

$$
\mathcal{F}_{\infty}=\{S \backslash K, K \text { is compact in } G\}
$$

Given $A \in \mathcal{F}_{\infty}$ and $\varepsilon>0$, an $(A, \varepsilon)$-chain is a finite sequence of operations consisting of applying an element of $A$ and followed by a jump of length at most $\varepsilon$. From this a $\mathcal{F}_{\infty}$-chain control set $E$ is defined in the same way as for control systems, by requiring that any two points in $E$ are linked by an $(A, \varepsilon)$-chain for any $A \in \mathcal{F}_{\infty}$ and $\varepsilon>0$. Since the elements of $\mathcal{F}_{\infty}$ are neighborhoods of $\infty$, the $\mathcal{F}_{\infty}$-chain control sets describe the asymptotic behavior of the $S$-action. 
The $\mathcal{F}_{\infty}$-chain control sets can be described by means of control sets. In fact, the equality

$$
E=\bigcap_{A, \varepsilon} D_{A, \varepsilon}
$$

holds, where $D_{A, \varepsilon}$ is a control set of the semigroup $S_{A, \varepsilon}$ generated by the $\varepsilon$ neighborhood of $A$ in $G$. This description permits us to carry over the theory outlined above for control sets in flag manifolds to $\mathcal{F}_{\infty}$-chain control sets. In particular, there is a subgroup of the Weyl group denoted by $W_{\mathcal{F}_{\infty}}(S)$, which gives the number of $\mathcal{F}_{\infty}$-chain control sets in the flag manifold $\mathbb{F}(1, \ldots d-1)$. Again, the invariant $\mathcal{F}_{\infty}$-chain control set (the one, which contains the invariant control set) contains all the information about $W_{\mathcal{F}_{\infty}}(S)$, and we can make the same comments and statements for the $\mathcal{F}_{\infty}$-chain control sets as well.

We expect that in a generic case the chain control sets on the full flag are just the closures of the main control sets (as for the situation on the projective space $\left.\mathbb{P}^{d-1}\right)$. Hence for this case the semigroup $S\left\{D_{w}: w \in W\right\}$ constructed above should also be the maximal one that has $\operatorname{cl} D_{w}, w \in W$, as the chain control sets.

Once we have a characterization of a semigroup from its transitivity and chain transitivity properties we can take into account the idea of bifurcation.

Problem 5.4 Study the bifurcation behavior of semigroups with respect to transitivity and chain transitivity.

The point here is to identify special classes of semigroups (bifurcation points) by imbedding them in a parametrized family of semigroups. From the point of view of dynamical systems or control systems these special semigroups should have a 'pathological' behavior, as explained in Section 2.

The following two examples may clarify this problem: Let $S=S^{0} \subset G$ be a maximal semigroup imbedded in a family $S^{\rho}$, for a parameter $\rho \in \mathbb{R}$. If this is an increasing family of semigroups then certainly $S^{0}$ is a bifurcation point of the family because of its maximality property.

On the other hand, let us consider the semigroups $S^{\rho} \in \mathrm{Gl}(d, \mathbb{R})$ coming from the bilinear control systems $\left(7^{\rho}\right), \rho \geq 0$.

For $S^{\rho}$ acting on the projective space $\mathbb{P}^{d-1}$ we know the following (compare [4]) under the inner pair condition (I):

(i) If for a certain $\rho$ there exists a main control set $D^{\rho}$ and a chain control set $E^{\rho}$ with $D^{\rho} \subset E^{\rho}$ and $E^{\rho} \neq \operatorname{cl} D^{\rho}$, then there is a second main control set $\tilde{D}^{\rho} \neq D^{\rho}$ with $\tilde{D}^{\rho} \subset E^{\rho}$. 
(ii) There are at most $d-1$ values of $\rho$ where the situation in (i) can occur. Let $\bar{\rho}>0$ be one of these exceptional values. Then $W\left(S^{\rho}\right) \neq W\left(S^{\rho^{\prime}}\right)$ for $\rho<\bar{\rho}<\rho$, and the map $\rho \mapsto \operatorname{cl} D^{\rho}$ is not continuous at $\bar{\rho}$ (in the Hausdorff topology over $\mathbb{P}^{d-1}$ ). Hence $\bar{\rho}$ can be considered as a bifurcation point of the transitivity behavior of the family $S^{\rho}, \rho \geq 0$, on $\mathbb{P}^{d-1}$.

In order to understand the behavior of semigroups on the full flag, one needs to show similar results for control sets and chain control sets on $\mathbb{F}(1, \ldots, d-1)$. We expect to get the following picture, under the assumption that $S^{\rho}, \rho>0$, has nonempty interior in $\mathrm{Sl}(d, \mathbb{R})$.

1. Each chain control set $E^{\rho}$ contains a main control set. So that if $W_{c}\left(S^{\rho}\right)$ stands for the subgroup of $W$ providing the chain control sets, then $W\left(S^{\rho}\right)$ is a subgroup of $W_{c}\left(S^{\rho}\right)$.

2. For fixed $w \in W$, the map $\rho \mapsto D_{w}^{\rho}$ is left continuous.

3. For fixed $w \in W$, the map $\rho \mapsto E_{w}^{\rho}$ is right continuous.

4. The discontinuity points of $\rho \mapsto W\left(S^{\rho}\right)$ are the continuity points of $D_{w}^{\rho}$.

5. If $\rho$ is a continuity point of $D_{w}(\cdot)$ then $E_{w}^{\rho}=\operatorname{cl} D_{w}^{\rho}$.

This picture is for the full flag in the maximal flag manifold. In another flag manifold discontinuity points should be a subset of the discontinuity points in the maximal flag. In particular, one has to check whether for some flag $\mathbb{F}^{\prime}$, there is a continuity point $\rho$ which is a discontinuity point for the full flag. This phenomena would show that the theory constructed in a smaller flag, e.g. the known one on $\mathbb{P}^{d-1}$, is not enough to get the full picture of the bifurcations of the control sets.

Apart from characterizing the discontinuity points of the (chain) control sets we also want to understand the transition of the semigroups through a discontinuity $\bar{\rho}$. The natural question here is: Given $S^{\rho}$ for $\rho<\bar{\rho}$, what are the possible characteristics of $S^{\rho}, \rho>\bar{\rho}$ ? In its simplest form, this would mean: Given $W\left(S^{\rho}\right)$ for $\rho<\bar{\rho}$, can $W\left(S^{\rho}\right)$ for $\rho>\bar{\rho}$ be an arbitrary subgroup of $W$ that contains $W\left(S^{\bar{\rho}}\right)$ ?

Up to now we have restricted ourselves to linear semigroups acting in a flag manifold. Equally important (for e.g. control systems and dynamical systems) is the action of a semigroup in a fiber bundle. As mentioned in the last section, this kind of semigroups shows up in the lifting of general nonlinear systems to the tangent bundle or some bundle obtained from it like the projective bundle or 
some flag bundle. We expect that once the linear situation is clarified it can be carried over to fiber bundles with the fiberwise techniques of [1].

As in Section 4. we stated the above problems for semigroups with nonempty interior in $\mathrm{Sl}(d, \mathbb{R})$. However, one should envisage the same theory for semigroups in a semi-simple Lie group $G$. Again, the boundaries of $G$ play the role of the classical flag manifolds and the Weyl group of $G$ substitutes the permutation group in $d$ elements.

\section{Asymptotic Behavior of Semigroups: The Radial Component}

The study of the dynamic behavior of semigroups has as its goal a classification theory. Such a classification could be algebraic (which will neeed different tools than the ones described here), based on transitivity and chain transitivity (compare Section 5.), or dynamic, i.e. topological. For linear dynamical systems a topological classification is available:

Two linear systems $\dot{x}=A x$ and $\dot{y}=B y$ in $\mathbb{R}^{d}$ are called topologically equivalent, if there exists a homeomorphism $h: \mathbb{R}^{d} \rightarrow \mathbb{R}^{d}$ that maps orbits into orbits, preserving the orientation. Hence equivalence is based on the flow of a system, allowing for time parametrization. It is well known (see e.g. Irwin [13, p. 86] or Robinson [17]) that two hyperbolic linear systems are topologically equivalent iff they have stable subpaces of the same dimension. Thus there are $d+1$ classes of linear hyperbolic systems on $\mathbb{R}^{d}$. Note that this characterization requires knowledge of the behavior of the compact component (dimension of the invariant subspaces) and of the radial component (positivity or negativity of the Lyapunov exponents which in this case are simply the real parts of the eigenvalues). The Grobman-Hartman Theorem generalizes the linear situation to nonlinear systems, locally around a singular point via linearization techniques.

A similar theory is available for random linear systems (see Cong [9]), which we formulate here in the context of bilinear control systems of the type (17) and their associated flows. Consider the function space $\mathcal{U}$ from (8) with shift $\Theta$ as defined in (9). Let $P$ be an ergodic invariant probability masure for the shift on $\mathcal{U}$. Let

$$
\dot{x}=A_{0} x+\sum_{i=1}^{m} u_{i}(t) A_{i} x \text { and } \dot{y}=B_{0} y+\sum_{i=1}^{m} u_{i}(t) B_{i} y
$$

be two bilinear systems in $\mathbb{R}^{d}$ with solutions $\varphi(t, x, u), \psi(t, y, u)$ and associated 
flows

$$
\Phi: \mathbb{R} \times \mathcal{U} \times \mathbb{R}^{d} \rightarrow \mathcal{U} \times \mathbb{R}^{d} \text { and } \Psi: \mathbb{R} \times \mathcal{U} \times \mathbb{R}^{d} \rightarrow \mathcal{U} \times \mathbb{R}^{d}
$$

respectively. A random homeomorphism is a measurable map

$$
h: \mathcal{U} \times \mathbb{R}^{d} \rightarrow \mathbb{R}^{d},(u, x) \mapsto h(u, x)
$$

such that for all $u \in \mathcal{U}$ the map $h(u)=h(u, \cdot): \mathbb{R}^{d} \rightarrow \mathbb{R}^{d}$ is a homeomorphism. The two flows $\Phi$ and $\Psi$ are called conjugate if there exists a random homeomorphism $h$ and a $\Theta$-invariant set $\mathcal{U}^{\prime}$ of full $P$-measure such that for all $u \in \mathcal{U}^{\prime}$ we have

$$
\Phi(t, u, \cdot)=h\left(\Theta_{t} u\right)^{-1} \circ \Psi(t, u, \cdot) \circ h(u) \quad \text { for all } t \in \mathbb{R}
$$

Hence, for the systems (30) the conjugacy $h$ maps trajectories into trajectories $P$ almost surely, but without time parametrization. (For hyperbolic random linear systems conjugacy and equivalence lead to the same criteria, since these flows admit no periodic points).

Cong's result says that two random linear systems are conjugate iff they have stable subspaces of the same dimension and they are both orientation preserving or orientation reversing on their stable subspaces with respect to a measurable choice of orientations. A similar theory for bilinear control systems, which would involve continuous homeomorphisms on $\mathcal{U} \times \mathbb{R}^{d}$, is not yet available.

We note that in the control theory literature, see e.g. Crouch [12], ideas for equivalence of control systems have been proposed based solely on equality of positive orbits. Here two bilinear control systems as in (30) are called equivalent if for all $x \in \mathbb{R}^{d}$ the corresponding orbits $\operatorname{cl}_{A}^{+}(x)=\operatorname{clO}_{B}^{+}(x)$ agree. This take into account only one of the aspects considered here and does not reduce to the dynamical systems concepts for control range $\rho=0$.

For more general semigroups we hope that a meaningful type of equivalence can be formulated when regarding their behavior on the compact component and the associated radial cocycles. Several ideas concerning the transitivity behavior on the compact component were described in the last section.

The study of the radial component presents new problems, because time is not an explicit parameter for a given semigroup. Hence the definition of Lyapunov exponents as in (20) has no obvious counterpart. Note, however, that the description of the Lyapunov exponents as in (20) allows for a description of the spectrum via ergodic theory as integrals of certain functions on the compact component with respect to invariant measures of the system, compare, e.g., [8]. Hence an ergodic theory for semigroup actions may provide approaches for this (and other problems). Finally, let us point out, that hyperbolicity for bilinear 
control systems can also be formulated in terms of controllability properties of the system in $\mathbb{R}^{d}$ (compare [7]), a condition that is easily generalized to general semigroups.

\section{References}

[1] C. Barros And L. SAn Martin, On the action of semigroups in fibre bundles.

[2] —, Chain control sets for semigroup actions, Comp. Appl. Math., 15 (1996), pp. 257-276.

[3] S. N. Chow and J. Hale, Methods of Bifurcation Theory, Springer-Verlag, 1982.

[4] F. Colonius and W. Kliemann, Linear control semigroups acting on projective space, J. Dyn. Diff. Equations, 5 (1993), pp. 495-528.

[5] — Some aspects of control systems as dynamical systems, J. Dyn. Diff. Equations, 5 (1993), pp. 469-494.

[6] - Limit behavior and genericity for nonlinear control systems, J. Diff. Equations, 109 (1994), pp. 8-41.

[7] — Asymptotic null controllability of bilinear systems, in Geometry in Nonlinear Control and Differential Inclusions, B. Jakubczyk and W. Respondek, eds., Banach Center Publications Vol. 32, Warsaw, 1995, pp. 139-148.

[8] — The Dynamics of Control, Birkhäuser, Boston, 2000.

[9] N. D. Cong, Structural stability and topological classification of continuous time linear hyperbolic cocycles, Random Comp. Dynamics, 5 (1997), pp. 19 63.

[10] C. Conley, Isolated Invariant Sets and the Morse Index, CBMS Regional Conference Series, No. 38, Amer. Math. Soc., 1978.

[11] W. Coppel, Dichotomies in Stability Theory, LN in Mathematics, Vol. 629, Springer-Verlag, 1978.

[12] P. E. Crouch, Lecture Notes on Geometric Non Linear Systems Theory. University of Warwick, Control Theory Center. 
[13] M. C. IRwin, Smooth Dynamical Systems, Academic Press, 1980.

[14] R. A. Johnson, K. J. Palmer, and G. R. Sell, Ergodic properties of linear dynamical systems, SIAM J. Math. Anal., 18 (1987), pp. 1-33.

[15] V. I. Oseledets, A multiplicative ergodic theorem. Lyapunov characteristic numbers for dynamical systems, Trans. Moscow Math. Soc., 19 (1968), pp. 197-231.

[16] J. Palis And W. DeMelo, Geometric Theory of Dynamical Systems: An Introduction, Springer-Verlag, 1982.

[17] C. Robinson, Dynamical Systems. Stability, Symbolic Dynamics, and Chaos, CRC Press, 1999. Second edition.

[18] D. Ruelle, Elements of Differentiable Dynamics and Bifurcation, Academic Press, 1989.

[19] R. J. Sacker and G. R. Sell, A spectral theory for linear differential systems, J. Diff. Equations, 27 (1978), pp. 320-358.

[20] S. Wiggins, Global Bifurcations and Chaos. Analytical Methods, SpringerVerlag, 1988. 